\section{Asociación de malformación anorrectal con duplicaciones anales y rectales}

\section{RESUMEN}

El objetivo de este trabajo es compartir la experiencia en el diagnóstico y tratamiento de casos de duplicaciones rectales y anales asociadas con malformación anorrectal.

Se reportan dos casos con oclusión intestinal y diagnóstico tardío de la malformación anorrectal tratados inicialmente con laparotomía, anastomosis latero-lateral de la duplicación colónica proximal y colostomía y un caso con diagnóstico de malformación anorrectal con fístula recto-perineal al nacimiento al que, al realizarle colostomía, evidenció duplicaciones colónica y rectal relacionadas. En los tres casos se realizó, para su corrección quirúrgica definitiva, un abordaje abdominoperineal y anastomosis latero-lateral de la duplicación rectal, colocación del recto dentro del complejo muscular anorrectal y posterior cierre de la colostomía. En un cuarto paciente con malformación anorrectal sin fístula clínicamente evidente y colostomía al nacimiento, la electroestimulación perineal evidenció dos complejos musculares a los que se les realizó un abordaje sagital, observándose dos fondos de sacos ciegos rectales separados. Se realizó anastomosis termino-lateral del recto duplicado y se decidió utilizar el complejo con mayor actividad contráctil para la colocación del neoano. Consideramos que el abordaje sagital posterior es la mejor opción quirúrgica para preservar el complejo muscular con mejor pronóstico para la continencia fecal.

Palabras claves: duplicación rectal, duplicación anal, malformación anorrectal.

\section{Association of anorectal malformation with anal and rectal duplication}

\section{ABSTRACT}

The objective of this paper is to report our experience in the diagnosis and therapy of rectal and anal duplications, all associated to anorectal malformation.

We present three cases of rectal duplications with anorectal malformation with recto-perineal fistula and colonic duplication. Two of them with delayed diagnosis and bowel obstruction, treated with laparotomy, colostomy and side-to-side anastomosis of the proximal colonic duplication; in the third case the diagnosis of the colonic and rectal duplication was made during a colostomy opening. For definitive correction, the
Karla A. Santos-Jasso ${ }^{1}$

Alejandro Ruíz-Montañez ${ }^{2}$

Esperanza Vidales-Nieto ${ }^{3}$

Luis de la Torre-Mondragón ${ }^{4}$

${ }^{1}$ Cirujana Pediatra Colorrectal. Clínica de Colon y Recto. Instituto Nacional de Pediatría. ${ }^{2}$ Cirujano Pediatra Colorrectal. Clínica de Colon y Recto. Hospital para el Niño Poblano.

${ }^{3}$ Residente de Cirugía Pediátrica.

${ }^{4}$ Profesor Titular del Curso Universitario de Alta Especialidad en Cirugía Pediátrica Colorrectal. Universidad Nacional Autónoma de México. Cirujano Pediatra. Clínica de Colon y Recto. Hospital para el Niño Poblano. Puebla, Puebla. México.

Recibido: 14 de noviembre 2013

Aceptado: 4 de abril 2014

Correspondencia: Dra. Karla A. Santos Jasso Instituto nacional de Pediatría Insurgentes Sur 3700-C CP. 04530, México D.F. Tel: (+52) 5510849000 ext. 1244 santosjasso@hotmail.com

Este artículo debe citarse como Santos-Jasso KA, Ruíz-Montañez A, Vidales-Nieto E, De la Torre-Mondragón L. Asociación de malformación anorrectal con duplicaciones anales y rectales. Acta Pediat Mex 2014;35:295-301. 
three patients underwent abdomino-perineal approach and side-to-side anastomosis of the rectal duplication, placement of the rectum within the muscle complex, and later on colostomy closure. In a fourth patient with anorectal malformation and colostomy after birth, the perineal electro-stimulation showed two muscle complexes. A posterior sagittal approach in both showed two separate blind rectal pouches; an endto-side anastomosis of the dilated rectum was made, and the muscle complex with stronger contraction was used for the anoplasty. The posterior sagittal approach is the best surgical option to preserve the muscle complex, with a better prognosis for rectal continence.

Keywords: Rectal duplication, anal duplication, anorectal malformations.

\section{ANTECEDENTES}

Las duplicaciones del tracto gastrointestinal son malformaciones congénitas poco frecuentes. Para que éstas se consideren verdaderas deben cumplir tres criterios: 1) localización continua, 2) tener dos capas de músculo liso y 3) mucosa de cualquier parte del tracto digestivo. ${ }^{1-3}$ El $5 \%$ del tracto intestinal corresponde al segmento rectal; ${ }^{1,4-6}$ sólo hay reporte de casos anecdóticos de las duplicaciones anales. ${ }^{7}$

Las duplicaciones rectales pueden tener presentación clínica y anatomía polimorfas: desde una masa en cavidad pélvica, la cual puede mostrar compresión al tracto urinario, un trayecto fistuloso al periné, hasta un prolapso rectal. ${ }^{5} \mathrm{Sin}$ embargo, la asociación de una duplicación anal con malformación anorrectal es un hallazgo menos frecuente. El objetivo de este trabajo es compartir la experiencia en el diagnóstico y tratamiento de cuatro casos de duplicación (rectal y anal) asociados con malformación anorrectal.

\section{CASO 1}

Niña de tres meses de edad que presentó obstrucción intestinal caracterizada por vómito biliar, distensión abdominal y ausencia de evacuaciones. A nivel perineal mostraba una malformación anorrectal con fístula recto-perineal y un tabique vaginal. (Figura 1) Se realizó laparotomía exploradora que mostró una duplicación del colon total tubular con gran dilatación del colon duplicado. Se efectuó comunicación de las estructuras duplicadas en forma proximal con sutura mecánica (engrapadora lineal cortante), hasta donde fue posible, y colostomía de dos bocas en la unión del colon descendente y sigmoides. La resonancia magnética pélvica mostró la dilatación del recto duplicado, la terminación en fondo de saco ciego de uno de los rectos y la presencia de una fístula al periné. (Figura 2) Al año de edad se efectuó abordaje mixto: 1) abdominal (comunicación distal del recto duplicado con engrapadoras lineales cortantes) y 2) sagital posterior, se encontró el término del saco ciego rectal duplicado a $2 \mathrm{~cm}$ del margen anal. Dos meses después la colostomía fue cerrada sin complicaciones.

\section{CASO 2}

Niña de siete años de edad con historia de estreñimiento y poca respuesta a tratamiento laxante. Se presentó al servicio de urgencias con 


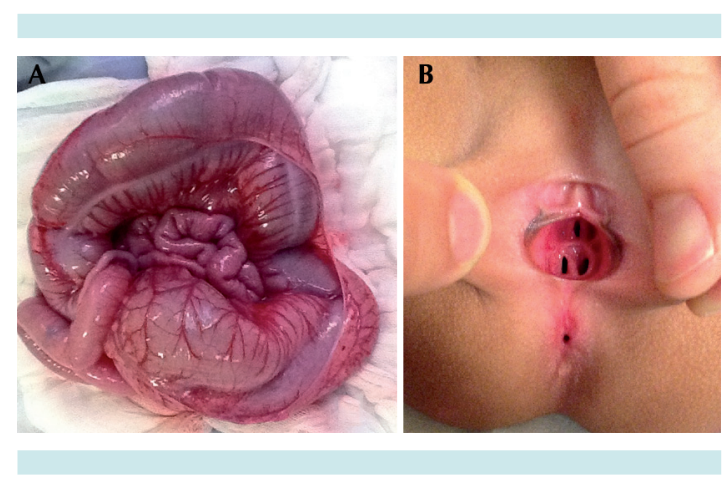

Figura 1. A) Laparotomía que muestra duplicación tubular del colon con gran dilatación. B) Periné que muestra la fístula rectoperineal y el tabique vaginal.

impactación fecal debida a la retención de una moneda en el recto. A la exploración física se le diagnosticó malformación anorrectal con fístula recto-perineal. El control radiológico mostró una imagen circular intrapélvica radioopaca. Se realizó rectoscopia para la extracción del cuerpo extraño. Se iniciaron irrigaciones rectales para desimpactación fecal. El colon por enema mostró megarrecto. Ya que la distensión abdominal no se resolvió, a pesar de la desimpactación fecal, se hizo laparotomía encontrándose duplicación colónica y rectal tubular que terminaba en fondo de saco ciego con gran dilatación quística terminal dentro del hueco pélvico, sin comunicación con el colon funcional. (Figura 3) Se realizó resección parcial del segmento distal duplicado y anastomosis termino-lateral al colon funcional, el cual terminaba en una fístula perineal. Se hizo una colostomía en ese mismo procedimiento quirúrgico. El reporte histopatológico reportó un espécimen de $48 \mathrm{~cm}$ de longitud y $8.5 \mathrm{~cm}$ de diámetro, al corte se identificó duplicación tubular de colon. En una segunda intervención se efectuó un abordaje sagital posterior para corrección de la malformación anorrectal y, finalmente, en una tercera, el cierre de la colostomía. No se determinaron otras malformaciones asociadas. A un año de seguimiento la niña presenta continencia fecal y un patrón regular de evacuaciones fecales.
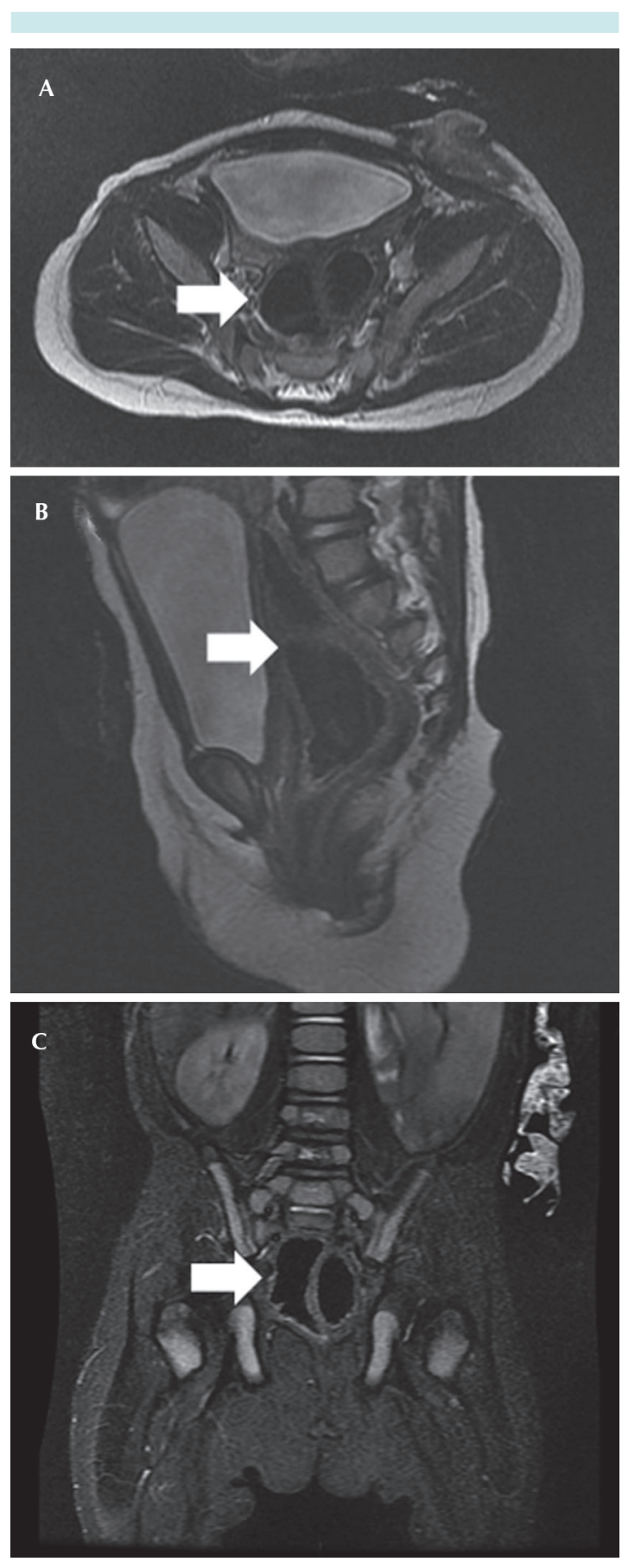

Figura 2. Resonancia magnética pélvica en cortes: $\mathbf{A}$ ) axial, B) sagital y C) coronal. La flecha muestra el sitio de la duplicación colónica. 


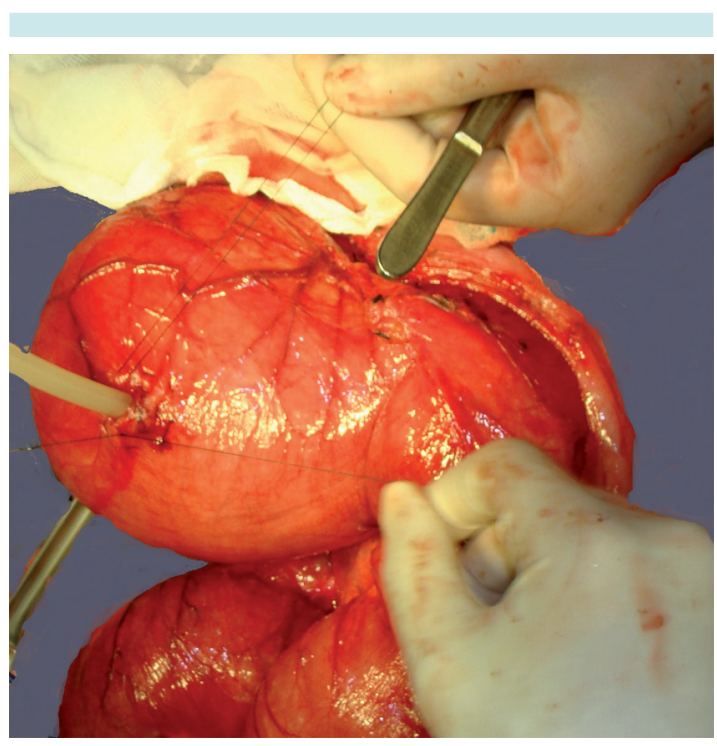

Figura 3. Laparotomía de una duplicación colónica tubular que terminaba en fondo de saco con gran dilatación quística.

\section{CASO 3}

Niño de un año de edad con diagnóstico, en la etapa neonatal, de malformación anorrectal con fístula recto-perineal, comunicación interventricular, hidronefrosis izquierda, hipospadias y microtia; al nacimiento se le realizó una colostomía de dos bocas. El colostograma mostró duplicación rectal tubular, terminando uno de los rectos en fondo de saco y el otro como una fístula perineal. (Figura 4) Se practicó anorrectoplastia sagital posterior encontrándose complejo muscular fuera de la línea media, desviado a la derecha y la duplicación rectal ya descrita por las imágenes del colostograma. Se hizo comunicación de la duplicación y anoplastia. Tres meses después se cerró la colostomía. A 6 meses de seguimiento no presenta estreñimiento y aún no hay control de esfínteres acorde con la edad actual.

\section{CASO 4}

Niño de un año de edad con malformación anorrectal sin fístula clínicamente evidente al

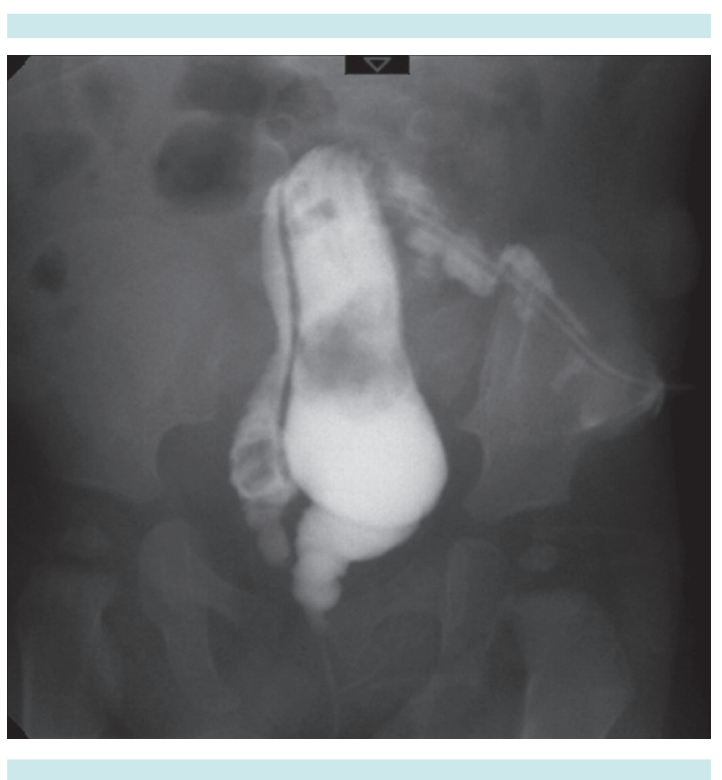

Figura 4. Colograma distal que muestra recto duplicado.

nacimiento. Se realizó colostomía al nacimiento. Durante la inspección perineal se encontraron dos fosetas anales reactivas a electroestimulación y dos orificios meatales urinarios funcionales. (Figura 5) Se efectuó un abordaje sagital posterior en ambos complejos musculares guiado por el electroestimulador y dividiéndolos a la mitad hasta la localización de dos fondos de saco rectales, se identificó el complejo muscular con mayor activad contráctil que fue elegido para el sitio final de la anoplastia. Se realizó una anastomosis del recto duplicado termino-lateral al recto que fue descendido, dejando un sólo complejo muscular funcional y anoplastia subsecuente. (Figura 6) A 4 años de seguimiento el niño tiene continencia fecal y un patrón normal de evacuaciones fecales.

\section{DISCUSIÓN}

A pesar de que la primera duplicación rectal fue descrita desde 1885 y la duplicación anal en $1956,{ }^{3}$ en la literatura actual sólo se han descrito experiencias anecdóticas. ${ }^{7}$ 


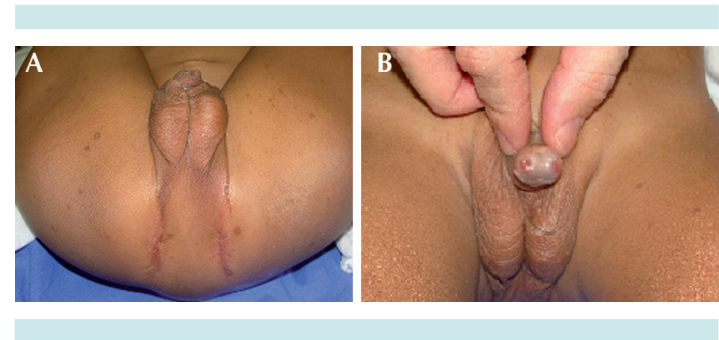

Figura 5. A) Región perineal que muestra ano imperforado sin fístula clínicamente evidente y dos complejos musculares. B) Imagen de uretra duplicada.

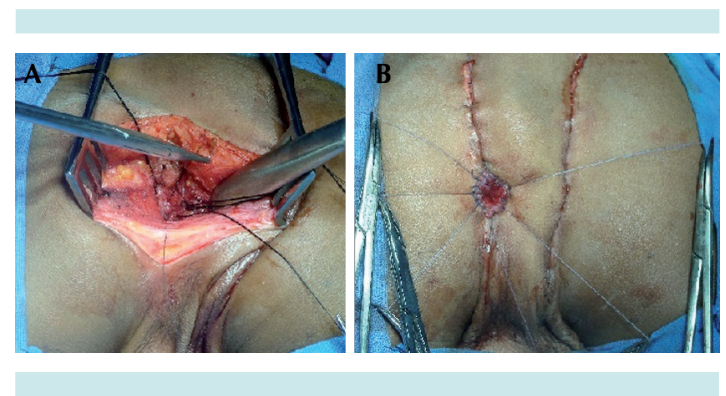

Figura 6. A) Anastomosis del recto duplicado terminolateral al recto que será descendido. B) Anoplastia.

Las bases embriológicas de las duplicaciones intestinales posteriores pueden ser explicadas por un trastorno en la diferenciación celular caudal en los días 23-25 de gestación, resultando una entidad en la que las estructuras derivadas de la notocorda y la cloaca son duplicadas en diferentes grados. ${ }^{5}$ La teoría más aceptada es la de Veeneklas, en la cual hay un defecto en la adherencia del endodermo a la notocorda; ;, 2,5,8 otras teorías son la propuesta por Lewis-Thyng (formación de divertículo en la semana 8-9 de gestación) y la teoría de la duplicación caudal. ${ }^{1-3,8-10}$

Suelen asociarse otras malformaciones: labio y paladar hendido, malrotación intestinal, cardiopatías, disgenesia lumbosacra, malformaciones urinarias (en $45 \%$ de los casos para duplicación anal) ${ }^{3}$, malformaciones renales en $60 \%$ de los casos, ${ }^{5}$ doble sistema colector, onfalocele, duplicación peneana, duplicación uretral (como es el caso de uno de nuestros pacientes); mielomeningocele, espina bífida oculta, ${ }^{1}$ teratoma, ependimoma, reflujo vesicoureteral y hemangiomas. ${ }^{11}$ Los pacientes que no presentan malformaciones espinales tienen mejor pronóstico. Tres de nuestros pacientes mostraron malformaciones asociadas: un caso con tabique vaginal; un caso con duplicación uretral; otro con hipospadias, hidronefrosis, microtia y cardiopatía; ninguno de nuestra serie tuvo disgenesia lumbosacra.

De acuerdo con Ladd y Gross ${ }^{12}$ una duplicación del tubo digestivo, en general, debe reunir tres criterios para que se considere como verdadera: 1) continuidad o contigüidad con el tubo digestivo normal, 2) tener dos capas musculares y 3) una mucosa intestinal normal. La mucosa ectópica gástrica o el tejido pancreático son raros.

Aunque no es una regla las duplicaciones rectales son frecuentemente posteriores y se pueden clasificar como: tipo I, quísticas y tipo II, tubulares. A su vez, las duplicaciones tipo I son las anomalías reportadas más comúnmente $(90 \%)$ y no tienen comunicación con el recto. La duplicaciones tipo II pueden ser divididas, a su vez, en aquellas que terminan en un fondo de saco ciego (II-A); las que comunican con el recto y presentan una fístula (II-B); las asociadas con fístulas que comunican hacia el periné (IIC) y quistes múltiples (II-D). Se han usado una variedad de términos para la duplicación rectal: enterocistoma del recto, quiste presacro, quiste rectal, hamartoma quístico.

La presentación clínica de la duplicación rectal depende de los siguientes factores: 1) el tamaño y el efecto de masa que ocasiona, 2) le existencia de fístula, 3) la sobreinfección de la duplicación, 4) la presencia de mucosa ectópica gástrica con ulceración y 5) degeneración maligna (adenocarcinoma). ${ }^{1,2,6}$ 
La mayoría de las duplicaciones rectales son localizadas en el espacio retrorrectal como una masa quística; ${ }^{5}$ sin embargo, diversas presentaciones clínicas pueden estar presentes, lo cual se corrobora claramente en nuestra serie de casos. En el periodo neonatal pocas veces son sintomáticas, a menos que se presenten como un abultamiento por fuera del canal anal o como un trayecto fistuloso que se abre en la línea media posterior. En los casos de duplicaciones quísticas localizadas en el área retrorrectal pueden manifestarse por compresión del recto y del tracto urinario. La duplicación quística rectal puede ser palpada al realizar un examen rectal, identificándose como una masa firme que protruye del hueco pélvico. Una duplicación quística, conforme incrementa de volumen por fluido acumulado, puede causar síntomas locales tales como: hipersensibilidad, dolor lumbar, dolor suprapúbico, obstrucción intestinal, disuria y dolor ciático (como sucedió en el caso dos de nuestra serie).

A su vez, la duplicación anal se puede presentar de manera aislada o como parte de un síndrome de duplicación caudal, asociándose de manera usual con duplicación rectal, colónica, o ambas. Existen tres formas clínicas de presentación de las duplicaciones anales: a) doble ano asociado con duplicaciones rectal y colónica, b) duplicación colónica con un ano normal y el otro ano imperforado con o sin fístula al tracto genitourinario y c) duplicación colónica, rectal y anal, con ambos anos imperforados con o sin fístula al tracto genitourinario (como fue el caso de uno de nuestros niños). La primera de éstas es en extremo rara y se puede localizar a los lados de la línea media. Los dos presentan complejo muscular independiente; sin embargo, uno de los dos está mejor desarrollado y la separación entre ellos es variable. Cuando se asocia con duplicación colónica ésta es usualmente tubular. La relación, de acuerdo con el género, favorece al femenino en proporción de 9:1, lo cual sugiere influencia de factores genéticos asociados; ${ }^{9} \sin$ embargo, se desconoce el mecanismo.

Las duplicaciones anales son definidas histopatológicamente por las siguientes características: 1) epitelio escamoso en el extremo caudal, 2) epitelio transicional en el extremo craneal y 3) músculo liso en la pared de la duplicación. ${ }^{3,4,6,8,11,13,14}$ Algunos autores reportan epitelio ciliar seudoestratificado de tipo respiratorio ${ }^{6}$ o células del epitelio vesical. ${ }^{4,13}$

Esta malformación se manifiesta por estreñimiento, secreción mucosa, fístulas recurrentes, prolapso rectal, dolor o inflamación, pero lo más común es que se detecte durante una inspección perineal. ${ }^{2-4,8,14,15} \mathrm{El}$ sangrado transrectal corresponde a una forma de presentación menos usual, normalmente asociado con mucosa ectópica gástrica, ${ }^{4,8,14}$ así como obstrucción urinaria e hidronefrosis en el periodo neonatal. $4,6,8$

Como apoyo para la elaboración del diagnóstico correcto de las duplicaciones rectales y anales se incluyen: radiografía de abdomen, ultrasonido, tomografía axial computada y resonancia magnética, siendo ésta última la más precisa para delimitar la localización, el volumen, las relaciones anatómicas del segmento duplicado, así como malformaciones asociadas. ${ }^{1-3,6,8,11,14,15}$

En el caso de las duplicaciones anales un enema contrastado o un cistograma miccional pueden ayudar a evidenciar fístula urinaria. Los estudios contrastados distales (colostogramas) destacaron por su utilidad en nuestra serie de casos.

Una vez realizado el diagnóstico el tratamiento debe ser quirúrgico y basar la elección en la localización y el tipo de duplicación. La resección total de la lesión previene la degeneración maligna de la duplicación; ;-3,8,10,13-15 bajo cualquier circunstancia se debe priorizar la continencia y la apariencia de los genitales externos. 
Santos-Jasso KA et al. Malformación anorrectal y duplicaciones anales y rectales

Las opciones disponibles son los abordajes sagital posterior, transanal, transcoccígeo y abordajes abdominales anteriores; el primero es el más utilizado ya que permite la corrección de fístulas si existen y mantiene intacta la anatomía de la continencia. ${ }^{1,6,10,14}$

El abordaje abdominal se utiliza en el caso de duplicaciones extensas y proximales, ${ }^{1,6,14}$ Ilegándose a complementar con un abordaje sagital posterior. Algunos autores prefieren únicamente la mucosectomía y la reparación primaria sin incidir sobre el complejo muscular anal previniendo, así, la lesión de éste y la transformación maligna; tiene la ventaja de ser un procedimiento más sencillo. ${ }^{15}$ Cuando la duplicación se encuentra separada por una membrana se puede realizar corte y anastomosis para formar un solo conducto utilizando engrapadoras mecánicas.

\section{REFERENCIAS}

1. Gupta DK, Sharma S. Rectal duplication and anal canal duplication. En: Holschneider AM. Hutson JM, editors. Anorectal Malformations in Children. Berlin: Springer; 2006, pp. 232-7.

2. Altinli E, Balkan T. Rectal Duplication as an Unusual Cause of Chronic Perianal Fistula in an Adult: Report of a case. Surgery Today 2004;34:796-8.

3. Narci A, Dilek FH, Centinkursun S. Anal canal duplication. Eur J Pediatr 2010;169:633-5.
4. Karaman I, Karaman A, Arda N, Cakmak O. External cystic rectal duplication: an unusual presentation of rectal duplication cyst. Singapore Med J 2007;48:287-8.

5. Ramírez RA, Asz J, Medina VF, Ortega SJ. Anterior colorectal duplication presenting as rectal prolapse. Pediatr Surg Int 2007;23:919-21.

6. Park WH, Choi SO, Park KK. Cystic rectal duplication: a rare cause of neonatal bladder-outlet obstruction and hydronephrosis. Pediatr Surg Int 2001;17:221-3.

7. Sinnya S, Curtis K, Walsh M, Wong D, Kimble R. Late presentation of anal canal duplication in an adolescent female-a rare diagnosis. Int J Colorectal Dis 2013;28:1175-6.

8. Marjanovic Z, Djordjevic I, Slavkiviv A, Krstic M. Rectal duplication, rare cause of constipation - case report. Cent Eur J Med 2012;7:621-3.

9. Koga J, Okazaki T, Kato Y, Lane GJ, Yamataka A. Anal canal duplication: experience at a single institution and literature review. Pediatr Surg Int 2010;26:985-8.

10. Delarue A, Garcia-Meric P, Guys JM. Antenatal rupture of a diverticular rectal duplication with neonatal perineal fistulization. Pediatr Surg Int 1998;13:288-9.

11. Lisi G, Illiceto MT, Rossi C. Anal Canal duplication a retrospective analysis of 12 cases from two European pediatric surgical departments. Pediatr Surg Int 2006;22:967-73.

12. Ladd WE, Gross RE. Surgical treatment of duplications of the alimentary tract. Surg Gynecol Obstet 1940;70:22145.

13. Craigie RJ, Abbaraju JS, Ba'ath ME, Turnock RR, Baillie CT. Anorectal malformation with tubular hindgut duplication. J Pediatr Surg 2006;41:e31-4.

14. Jackson KL, Peche WJ, Rollins MD. An unusual presentation of a rectal duplication cyst. Int I Surg Case Rep 2012;3:3145.

15. Tiryaki T, Senel E, Atayurt H. Anal Canal duplication in children: A new technique. Pediatr Surg Int 2006;22:560-1. 